\title{
Surface imaging of stellar non-radial pulsations
}

\section{Inversions of simulated data}

\author{
S. V. Berdyugina ${ }^{1,2}$, J. H. Telting ${ }^{3}$, and H. Korhonen ${ }^{4}$
}

1 Astronomy Division, University of Oulu, PO Box 3000, 90401 Oulu, Finland
2 Institute of Astronomy, ETHZ, 8092 Zurich, Switzerland
3 Nordic Optical Telescope, Apartado 474, 38700 S/C de La Palma, Canarias, Spain
${ }^{4}$ Astrophysical Institute Potsdam, An der Sternwarte 16, 14482 Potsdam, Germany

Received 10 February 2003 / Accepted 13 May 2003

\begin{abstract}
We investigate capability of the stellar surface imaging technique for studying stellar non-radial pulsations on the basis of inversions of time series of variable line profiles without making assumptions on the specific shape of the pulsations. The inversion results in an image of the stellar surface in which sectoral and tesseral modes can be distinguished in many cases, and the pulsational degree $\ell$ and the azimuthal order $|m|$ can be determined. We find that sectoral and tesseral modes with $\ell-|m|=2$ (or any even number) can be successfully restored under various conditions (different inclinations of the rotational axis, surface temperature or velocity fluctuations due to pulsations). Tesseral modes with $\ell-|m|=1$ (or any odd number) do not show significant line profile variations at higher inclinations of the rotational axis, and, thus, no information can be recovered from line profiles. At lower inclinations, only the azimuthal order $|m|$ of such modes can be recovered. We conclude that stellar surface imaging is a useful technique for pulsation-mode identification.
\end{abstract}

Key words. stars: imaging - stars: oscillations

\section{Introduction}

Internal structure of stars can be studied by means of asteroseismology, i.e. by observing and analysis of stellar oscillations. Observed oscillation frequencies depend on the properties of the star and, thus, constrain the stellar structure and evolution models. Furthermore, it is important to identify oscillation modes, namely to assign a pulsational degree and azimuthal number to the observed frequency. Recent reviews on pulsation-mode identification methods are presented by Aerts \& Eyer (2000), Balona (2000), Mantegazza (2000), and Telting (2003).

Stellar non-radial pulsations (NRP) on rapidly rotating stars are observed spectroscopically as systematic line-profile variations $(l p v)$. The pulsations re-distribute the flux in a rotationally broadened profile due to combined effects of the pulsational velocity field and temperature fluctuations. With the Fourier analysis of time series of profiles, dominant frequencies of pulsations can be found. Then, the pulsational degree $\ell$, the azimuthal order $m$ and the velocity amplitude of the pulsations can be derived with an appropriate data set (Gies \& Kullavanijaya 1988; Schrijvers et al. 1997; Telting \& Schrijvers 1997; Schrijvers \& Telting 1999). The difficulties arise when the value of $|m|$ is determined. It is generally

Send offprint requests to: S. V. Berdyugina,

e-mail: sveta@astro.phys.ethz.ch assumed that $\ell=|m|$, i.e. that the pulsation modes are sectoral. However, Telting \& Schrijvers (1997) showed that in some cases the value of $|m|$ can be estimated from line profiles.

A great help in the identification of the pulsation modes can be provided by the technique of stellar surface imaging based on inversions of a time series of line profiles into an instant stellar image. The idea of surface imaging (or Doppler imaging) was invented by Deutsch (1958) and was first used to map chemical inhomogeneities on Ap stars. The further development of the method by Deutsch (1970), Falk \& Wehlau (1974), Goncharsky et al. (1977) and many others has lead to a fully functional method for mapping stellar surface structures. Surface imaging is an ill-posed inversion problem, and many different methods have been used for solving it, e.g., Maximum Entropy Method (Vogt \& Penrod 1983; Vogt et al. 1987), Tikhonov Regularization (Goncharsky et al. 1977; Piskunov et al. 1990) and Occamian Approach (Berdyugina 1998). Nowadays, surface imaging is widely used for mapping temperature, abundance and magnetic field fluctuations on the surfaces of magnetically active, rapidly rotating hot Ap stars and cool G-K-M stars.

In this paper, we study how surface imaging technique can be used for mapping stellar NRP. We perform inversions of theoretical line profiles calculated for various pulsation modes and show that the inversions result in images of the stellar surface in which sectoral and tesseral modes can be distinguished. Our 
Table 1. Calculated models of NRP for the test star pulsating with the frequency 15 cycle/day.

\begin{tabular}{rrrrl}
\hline \hline Set & $\ell$ & $|m|$ & $i$ & \multicolumn{1}{c}{ Fluctuation } \\
\hline \multicolumn{5}{c}{$P_{\mathrm{r}}=6.4 \mathrm{~h}$} \\
1 & 4 & 4 & $60^{\circ}$ & $\Delta T / T=10 \%$ \\
\hline \multicolumn{4}{c}{$P_{\mathrm{r}}=14.4 \mathrm{~h}$} \\
2 & 9 & 9 & $60^{\circ}$ & $\Delta T / T=10 \%$ \\
3 & 9 & 9 & $60^{\circ}$ & $\Delta v=3 \mathrm{~km} \mathrm{~s}^{-1}$ \\
4 & 9 & 9 & $60^{\circ}$ & $\Delta v=10 \mathrm{~km} \mathrm{~s}^{-1}$ \\
\hline \multicolumn{4}{c}{$P_{\mathrm{r}}=4.8 \mathrm{~h}$} \\
5 & 5 & 3 & $55^{\circ}$ & $\Delta T / T=10 \%$ \\
6 & 5 & 3 & $70^{\circ}$ & $\Delta T / T=10 \%$ \\
7 & 5 & 3 & $85^{\circ}$ & $\Delta T / T=10 \%$ \\
8 & 5 & 3 & $55^{\circ}$ & $\Delta v=10 \mathrm{~km} \mathrm{~s}^{-1}$ \\
9 & 5 & 3 & $70^{\circ}$ & $\Delta v=10 \mathrm{~km} \mathrm{~s}^{-1}$ \\
10 & 5 & 3 & $85^{\circ}$ & $\Delta v=10 \mathrm{~km} \mathrm{~s}^{-1}$ \\
\hline \multicolumn{4}{c}{$P_{\mathrm{r}}=6.4 \mathrm{~h}$} \\
11 & 5 & 4 & $55^{\circ}$ & $\Delta T / T=10 \%$ \\
12 & 5 & 4 & $85^{\circ}$ & $\Delta T / T=10 \%$ \\
13 & 5 & 4 & $55^{\circ}$ & $\Delta v=10 \mathrm{~km} \mathrm{~s}^{-1}$ \\
14 & 5 & 4 & $85^{\circ}$ & $\Delta v=10 \mathrm{~km} \mathrm{~s}^{-1}$ \\
\hline & &
\end{tabular}

results apply to $\mathrm{p}$-mode oscillations in rotating $\beta$ Cephei and $\delta$ Scuti variables.

\section{Simulated data}

We calculated 14 sets of profiles of the Si III $4552 \AA$ line for a test star with the following parameters: $T_{\text {eff }}=25000 \mathrm{~K}$, $\log g=4.0, v \sin i=100 \mathrm{~km} \mathrm{~s}^{-1}$. The time series of simulated spectra were generated with the model presented by Telting \& Schrijvers (1997) and Schrijvers \& Telting (1999). In different sets, the test star is assumed to pulsate in different modes, sectoral or tesseral, with either temperature or velocity fluctuations. This is done in order to demonstrate how the method works for different $\ell$ and $|m|$ of NRP. For instance, the following cases are of particular interest: (i) sectoral mode implying $\ell=|m|$; (ii) tesseral mode with $\ell-|m|=2$ which is symmetric with respect to the equator; (iii) tesseral mode with $\ell-|m|=1$ which is antisymmetric with respect to the equator as it has one node line coinciding with the equator. Also, by choosing different values of the inclination of the stellar rotation axis, $i$, we test the sensitivity of the solution to this parameter. A summary of the calculated models is given in Table 1. We assume that the test star oscillates with the frequency 15 cycles/day (in the observers' frame) in all pulsation modes. This results, however, in different pattern-rotation periods $P_{\mathrm{r}}$, which are given in Table 1 . The line profiles were simulated with $S / N=500$ for 22 rotational phases covering a whole rotation of a single pulsation.

\section{The inversion technique}

To perform inversions of the simulated data and recover the pulsation patterns, we assume for simplicity that the $l p v$ are caused only by temperature fluctuations, despite the fact that the simulations included also velocity variations.

For a rapidly-rotating star the rotational broadening of line profiles is significantly larger than the local line profile at a single point on the stellar surface. Therefore, a temperature or velocity fluctuation on the stellar surface results in a "bump" in the profile. As the pulsation pattern rotates, this "bump" moves across the profile over a velocity range that depends on the latitude of the fluctuation (see e.g. Vogt \& Penrod 1983). Inversion of a time series of stellar line profiles results in a map, or image, of the stellar surface.

Given a set of stellar atmosphere models, the stellar image reflects the distribution of effective temperatures of the models across the stellar surface, as usual for the surface imaging technique. The stellar surface is considered as a set of elemental areas, $\mathbf{S}$, where the temperature values have to be determined from the observational data set $\mathbf{D}$. To achieve this, we solve the equation

$\mathbf{D}=\mathbf{P S}$

where $\mathbf{P}$ denotes the Point Spread Function (PSF), or the response operator, which determines the transformation of the image to the data, or the model. Elements of the matrix $\mathbf{P}$ are proportional to local line profiles and local areas.

A set of local line profiles have been calculated for a temperature range of $20000-30000 \mathrm{~K}$ and $\log g=4.0$ using the stellar atmosphere models by Kurucz (1993). A code used for synthetic spectrum calculations is described in detail by Berdyugina (1991). It includes calculation of opacities, intensities and fluxes in the continuum and lines. In order to take into account limb darkening effect, the local line profiles have been calculated for ten angular points on the stellar disk. The stellar surface was divided by the coordinate grid of $3^{\circ} \times 3^{\circ}$, and the temperature of each pixel was recovered in the inversion.

Since the surface imaging problem is a typical inverse problem, which tries to determine the true properties of the phenomena from the observed effects, a special method must be used to solve it. Here, we use the Occamian approach to inverse problems which was applied to the surface imaging problem by Berdyugina (1998). With simulated data and test calculations it was shown that the Occamian approach can restore stellar images under various conditions. The method was also successfully used for temperature mapping of cool stars (see e.g. Berdyugina et al. 1998, 1999).

Note that our inversion technique is free from assumptions that it is the pulsations that cause line profile variations. In contrast with many other techniques, we do not assume that the surface-feature distribution is to be in the form of spherical harmonics or any other presupposed shape. This provides an opportunity to test with real data if such an assumption made in previous studies is reliable. Also, we do not make any assumptions if pulsation modes are of sectoral or tesseral type. 


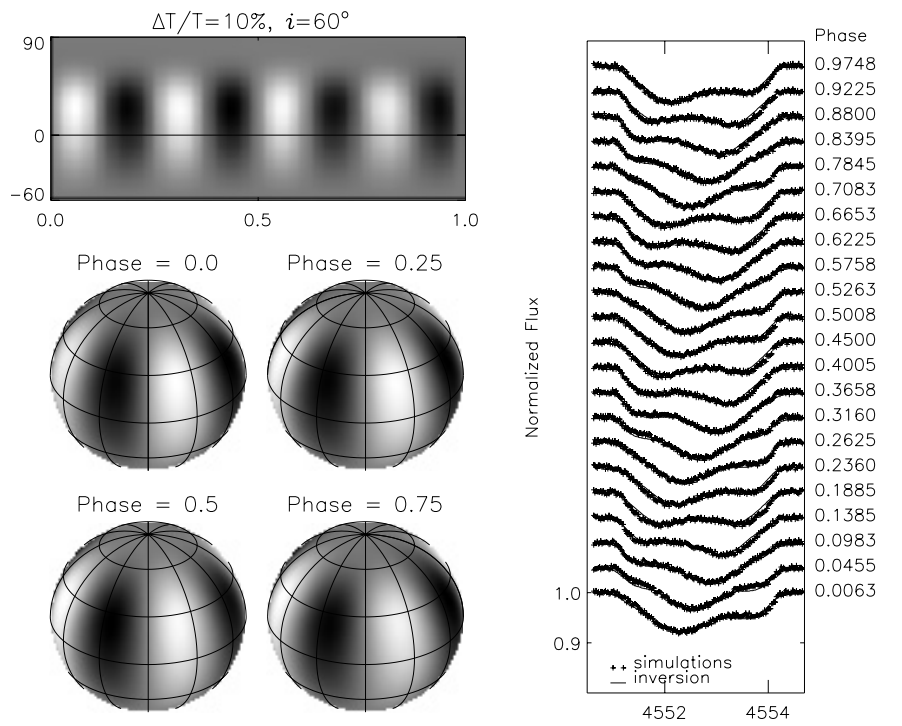

Fig. 1. Inversion results for the sectoral mode $\ell=|m|=4$ with only temperature fluctuations (Set 1). The image is shown in the cylindrical and spherical projection. Because of significant inclination of the stellar rotation axis, the pulsations are not completely recovered in the subequatorial zone.

\section{Inversions with known parameters}

\subsection{Sectoral modes}

For sectoral modes we considered two cases with temperature fluctuations of $10 \%: \ell=|m|=4$ and $\ell=|m|=9$. The latter case was also studied for two values of velocity fluctuations: 3 and $10 \mathrm{~km} \mathrm{~s}^{-1}$. The inversion results are shown in Figs. 1 and 2 .

In all cases, the recovered images of the test star show a distinct sectoral structure, in good agreement with the input pulsation patterns. Because of significant inclination of the rotational axis $\left(i=60^{\circ}\right)$, the pulsations are not completely recovered in the subequatorial zone and, thus, look as shifted in latitude. This, however, does not disturb the mode identification. The amplitude of temperature fluctuations is restored reasonably well.

The velocity fluctuations interpreted in terms of temperature fluctuations also result in well-defined pulsation patterns. The shapes of bumps in the images correspond well to the shapes of bumps in spectra. Particularly, in the case of velocity fluctuations of $10 \mathrm{~km} \mathrm{~s}^{-1}$, dips in the line profiles, being narrower than the bumps, result in narrow bright regions in the image (the bottom image in Fig. 2), while broader dark regions correspond to the line profile bumps. Such shapes, if observed, can therefore serve as a signature of strong velocity fields in the pulsations.

\subsection{Symmetric tesseral mode}

The inversion results for the tesseral mode $\ell=5$ and $|m|=3$ with temperature fluctuations of $10 \%$ and velocity fluctuations of $10 \mathrm{~km} \mathrm{~s}^{-1}$ are shown in Figs. 3 and 4, respectively. Such a mode consists of three rows of a tesseral pattern which is symmetrical with respect to the equator. The two cases have been
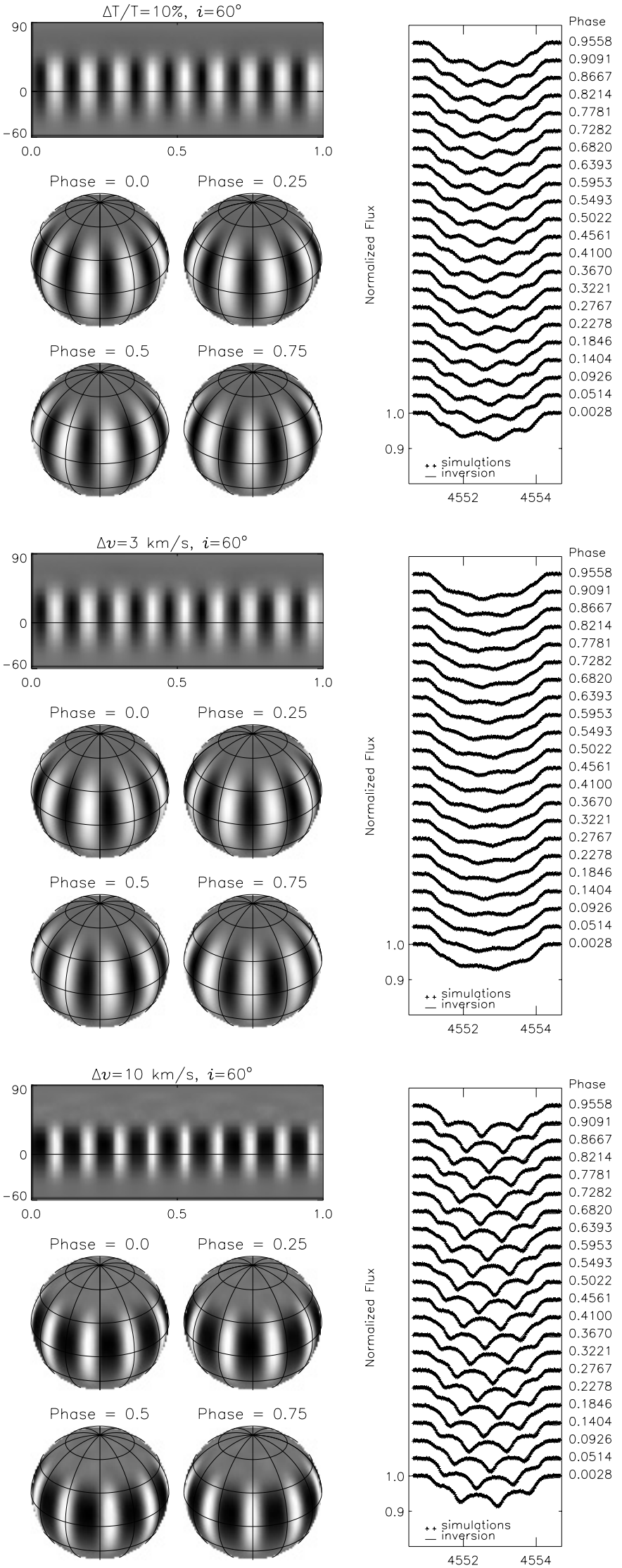

Fig. 2. Inversion results for the sectoral mode $\ell=|m|=9$ with temperature and velocity fluctuations (Sets 2-4). 
S. V. Berdyugina et al.: Surface imaging of stellar non-radial pulsations. I.
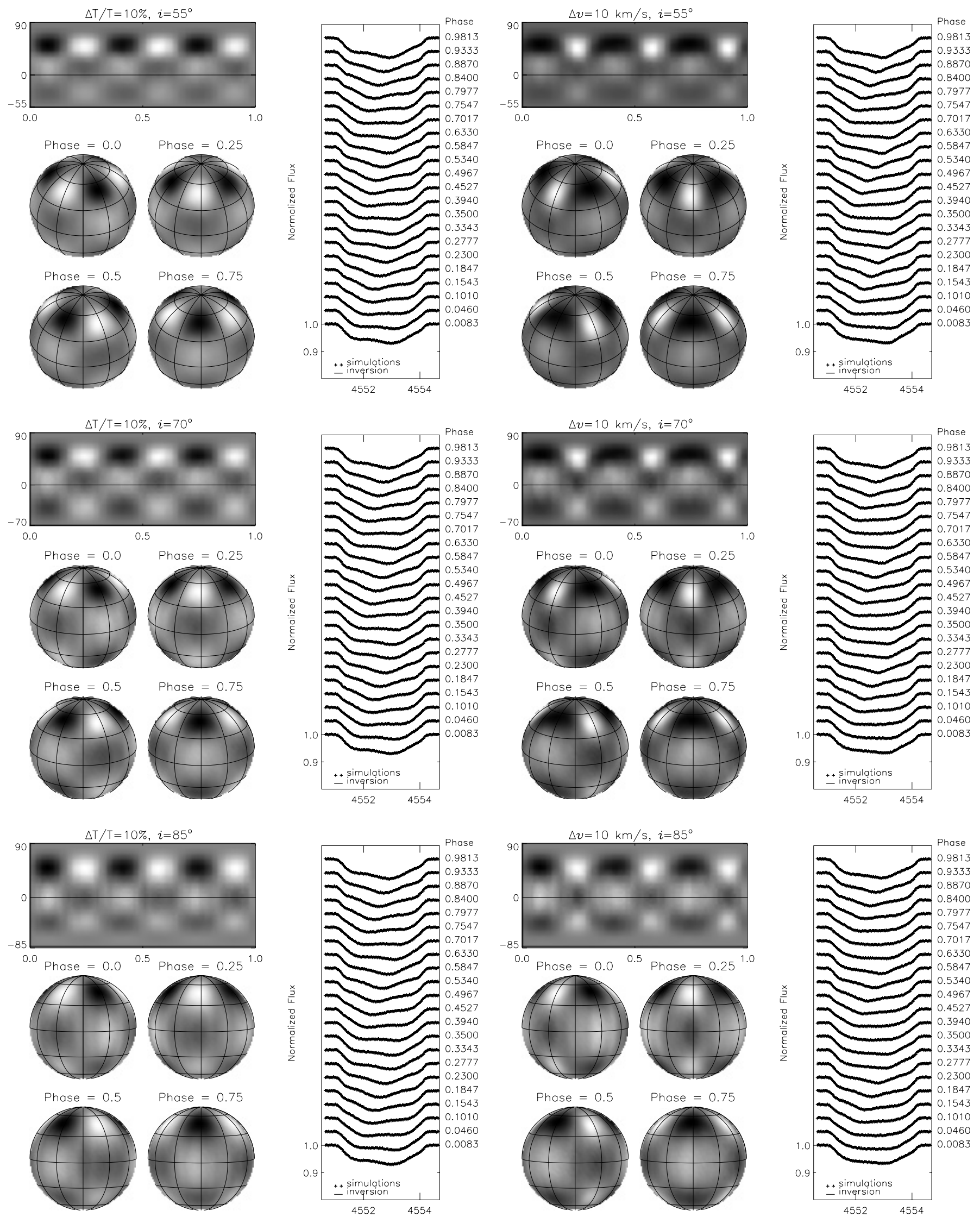

Fig. 3. Inversion results for the symmetrical tesseral mode $\ell=5,|m|=$ Fig. 4. The same as Fig. 3 for velocity fluctuations (Sets 8-10). 3 with temperature fluctuations at different inclinations (Sets 5-7). 

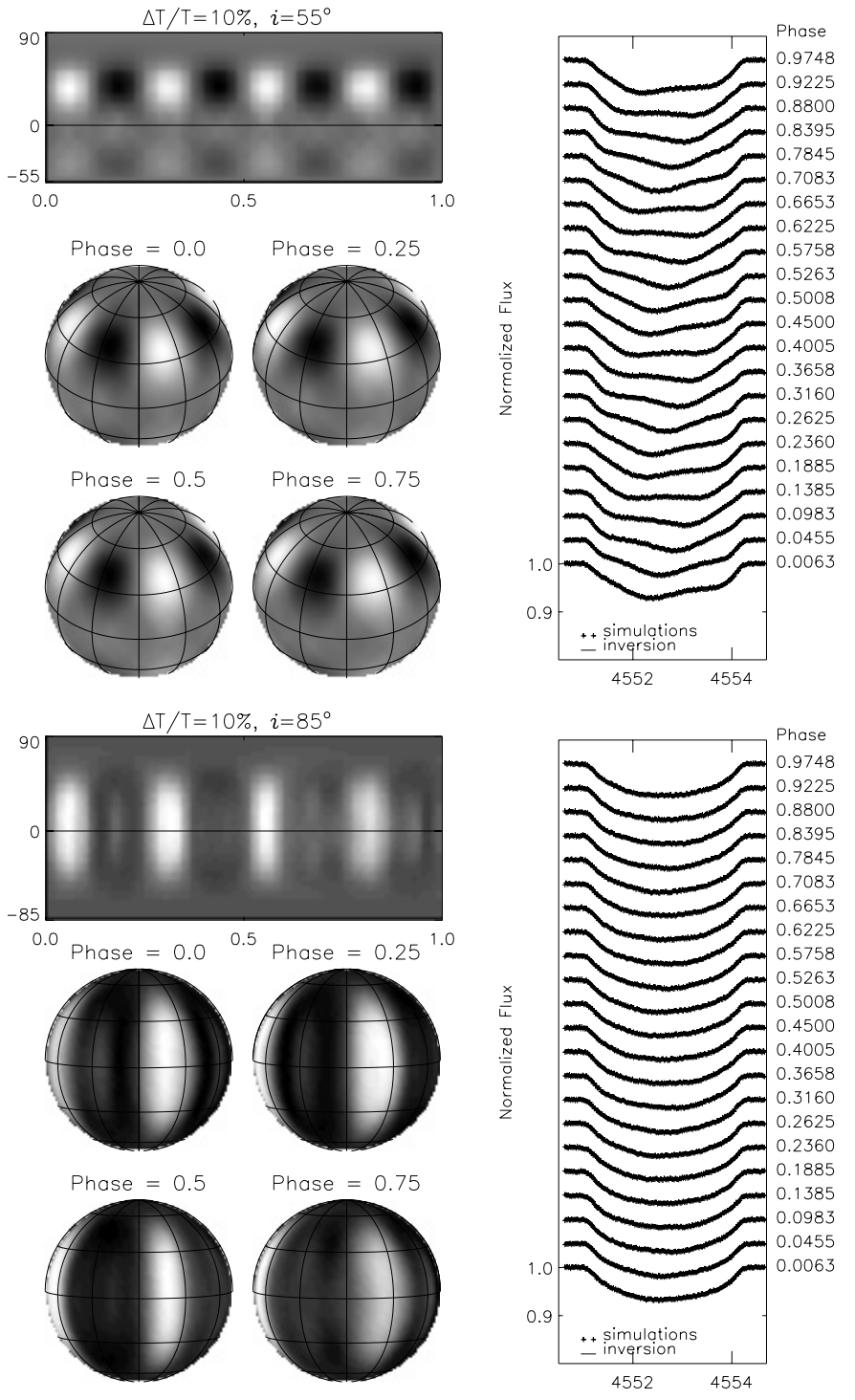

Fig. 5. Inversion results for the antisymmetric tesseral mode $\ell=$ $5,|m|=4$ with temperature fluctuations at different inclinations (Sets 11-12).

tested for three different inclinations of the rotation axis to the line of sight: $55^{\circ}, 70^{\circ}$, and $85^{\circ}$.

The recovered images again show rather distinct structures in good agreement with the input pulsation patterns. At lower inclination, the subequatorial zone is not well recovered and high-latitude structures dominate the image. Also, as was obtained for sectoral modes, the structures look shifted to higher latitudes at lower inclinations, while this effect is completely gone at the inclination of $85^{\circ}$.

For temperature fluctuations, light and dark regions in the images as well as corresponding dips and bumps in line profiles are of about the same size and shape. For velocity fluctuations, bumps are noticeably broader than dips in line profiles, that results in more extended dark regions in the recovered images. This again may serve as an indication of strong prevailing velocity fields in pulsations, if observed (see also Schrijvers et al. 1997).
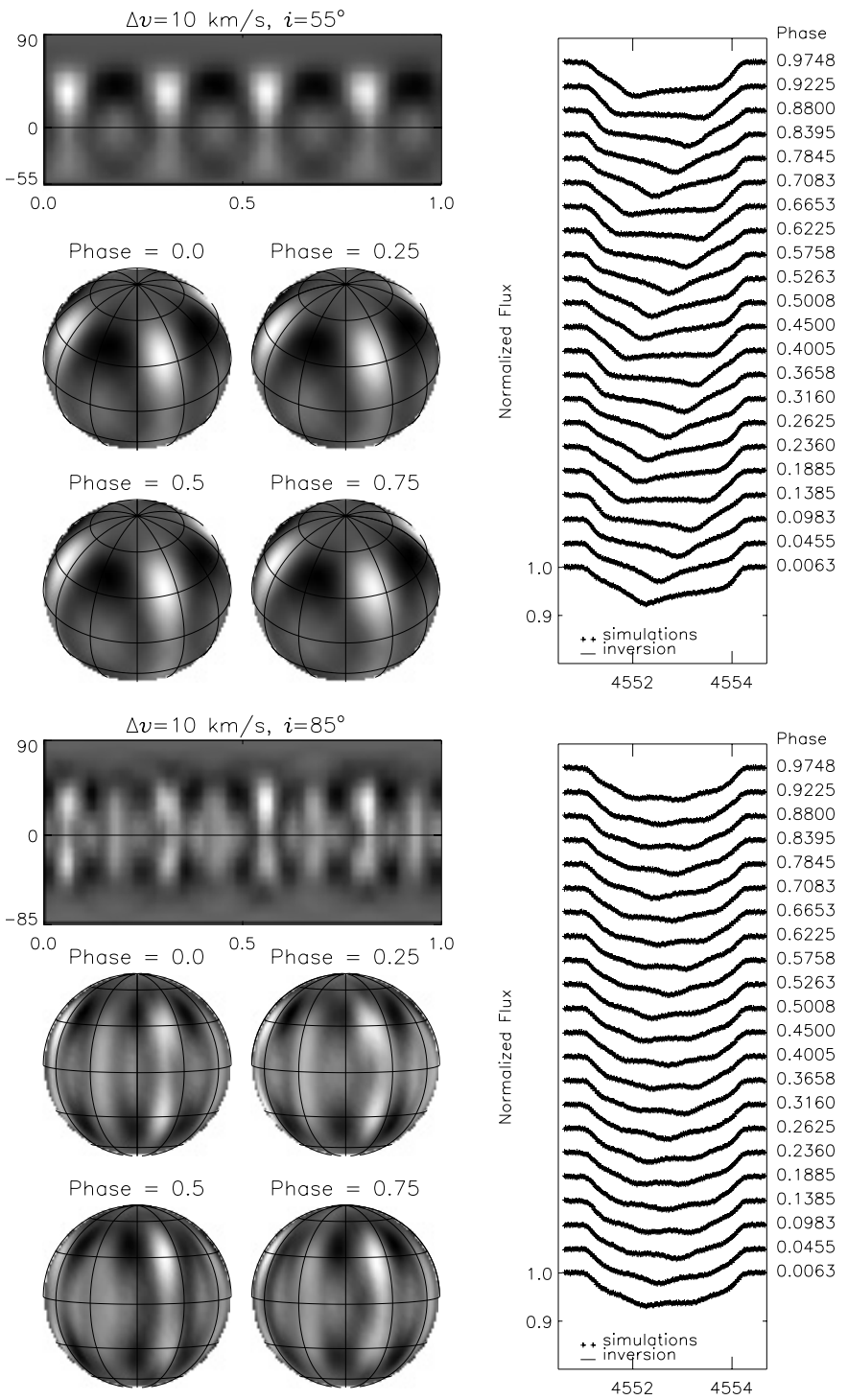

Fig. 6. The same as Fig. 5 for velocity fluctuations (Sets 13-14).

\subsection{Antisymmetric tesseral mode}

The inversion results for the tesseral mode $\ell=5$ and $|m|=4$ with temperature fluctuations of $10 \%$ and velocity fluctuations of $10 \mathrm{~km} \mathrm{~s}^{-1}$ are shown in Figs. 5 and 6, respectively. Such a mode consist of two rows of tesseral patterns which are antisymmetric with respect to the equator. The two cases have been tested for two different inclinations of the rotation axis to the line of sight: $55^{\circ}$ and $85^{\circ}$.

We found that this is a rather difficult case. For instance, if the rotation axis is perpendicular to the line of sight, Doppler shifts in either side of the stellar equator are equivalent. Further, if temperature or velocity fluctuations at the same Doppler shift on different sides of the equator are of opposite signs, as it occurs in case of antisymmetric tesseral modes, the resulted signal in the line profile at this Doppler shift will stay unchanged, as there would be no fluctuations at all. Therefore, line profile variations are rather small for these modes at higher inclinations (see bottom line profiles in Figs. 5 and 6), and the inversion procedure cannot deduce true pulsation patterns. 
Relatively good results can be achieved at lower inclinations, as seen from the top plots in Figs. 5 and 6, especially for temperature fluctuations. The structure recovered at $i=55^{\circ}$ clearly indicates the azimuthal number $|m|=4$. However, because the surface imaging technique is based on the analysis of Doppler shifts of local line profiles, the procedure cannot distinguish the equivalent shifts on different sides of the stellar equator and produces the known effect of reflection of recovered structures with respect to the equator. It is especially noticeable in case of antisymmetric pulsation patterns, while it is successfully masked in case of symmetric modes. Its appearance, therefore, may provide some hints for the mode to be antisymmetric.

\section{Influence of parameter errors}

For obtaining reasonable results from the inversions, certain requirements have to be fulfilled. First, observed spectra should be of high resolution $(\lambda / \Delta \lambda \geq 30000)$ and high signal-to-noise ratio $(S / N \geq 200)$. Chosen spectral lines should be sensitive to NRP and show significant line profile variations. It is also preferable if they are not blended. The rotation of the star must be fast enough to use the technique $\left(v \sin i \geq 20 \mathrm{~km} \mathrm{~s}^{-1}\right)$. The spectra should cover at least one full pattern-rotation period with good time resolution. More rotations are needed for obtaining a reliable frequency of NRP. Further, the spectra should be reduced to exclude systematic radial velocity variations, e.g., due to orbital motion in binary systems.

The second group of requirements concerns a set of input geometrical and physical parameters which are needed for synthetic spectra calculations. The geometrical parameters are the inclination of the rotational axis to the line of sight $i$, projection of the equatorial rotational velocity $v \sin i$, and observed oscillation frequency $f$ or pattern-rotation period. The physical parameters are the effective temperature of the star $T_{\text {eff }}$, surface gravity $\log g$, micro- and macroturbulent velocities. The accurate physical parameters are important for deducing physical properties of the pulsations, such as amplitudes of temperature and velocity fluctuations on the stellar surface. For the mode identification purpose, however, these parameters are not crucial and should only result in a good fit to the average observed line profile. Moreover, the stellar effective temperature is not fixed in inversions and can be fine-tuned by iterations. Synthetic spectra calculations should account for limb darkening of the stellar disk and for blends, including possible contribution from a companion in binary systems.

The most critical stellar parameters for the NRP mode identification are the geometrical parameters. While $v \sin i$ and $f$ can be directly deduced from spectral observations with good accuracy, the inclination angle $i$ can be only estimated with high uncertainty. In this section, we investigate the influence of errors in these parameters on the result of inversions. Some tests for uneven cool spot distribution with the present inversion method, including effects of low $S / N$ and errors in $T_{\text {eff }}, v \sin i$ and $i$, can be also found in the paper by Berdyugina (1998).

For a sample of parameter errors we carried out inversions for two sets of simulations: sectoral mode with $\ell=|m|=9$ (Set 2) and tesseral mode with $\ell=5,|m|=3$ (Set 6).
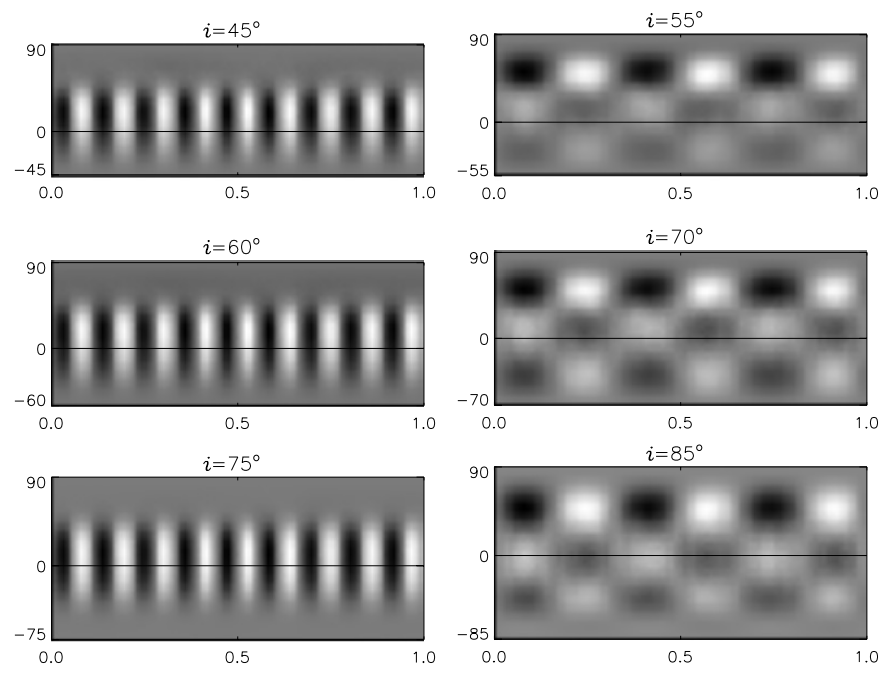

Fig. 7. The images of the Sets 2 and 6 (left and right) recovered with the true inclination of the rotational axis of $60^{\circ}$ and $70^{\circ}$, respectively, and pairs of values deviating from those by $\pm 15^{\circ}$.

\subsection{Inclination}

Originally, Sets 2 and 6 have been calculated for the inclination angles $i$ of $60^{\circ}$ and $70^{\circ}$ respectively (Table 1). To estimate the effect of inclination errors, which can be quite large, we allowed the inclination angle to vary by $\pm 15^{\circ}$ for both sets. The results are shown in Fig. 7.

In all cases, the recovered images of the test star show distinct pulsational patterns, in good agreement with the input images. There is no noticeable changes in the structures except small latitudinal shifts, which occur because of changes of the sub-observed latitude on the stellar disk.

We conclude that errors in the inclination angle do not significantly effect the recovered pulsation pattern and, thus, will not influence the mode identification.

\subsection{Rotational velocity}

To estimate the effect of rotational velocity errors we allowed it to vary by $\pm 5 \mathrm{~km} \mathrm{~s}^{-1}$ for both sets. The results are shown in Fig. 8. As was known from previously published tests (Berdyugina 1998), underestimating of the rotational velocity results in darkening (cooling) the polar regions, while overestimating results in their brightening. This effect is very well seen in the images for the sectoral mode (left column in Fig. 8). In case of the tesseral mode (right column in Fig. 8), the effect of smaller rotational velocity is seen in the enhancement of the dark patterns near the pole and of the bright patterns near the equator. The opposite behaviour is found for the case when the rotational velocity is overestimated.

We conclude that errors in the rotational velocity influence significantly the contrast of the recovered pattern in the latitudinal direction, while they do not influence the mode identification. 


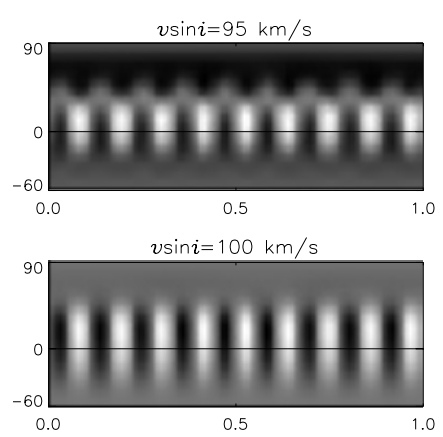

$v \sin i=105 \mathrm{~km} / \mathrm{s}$
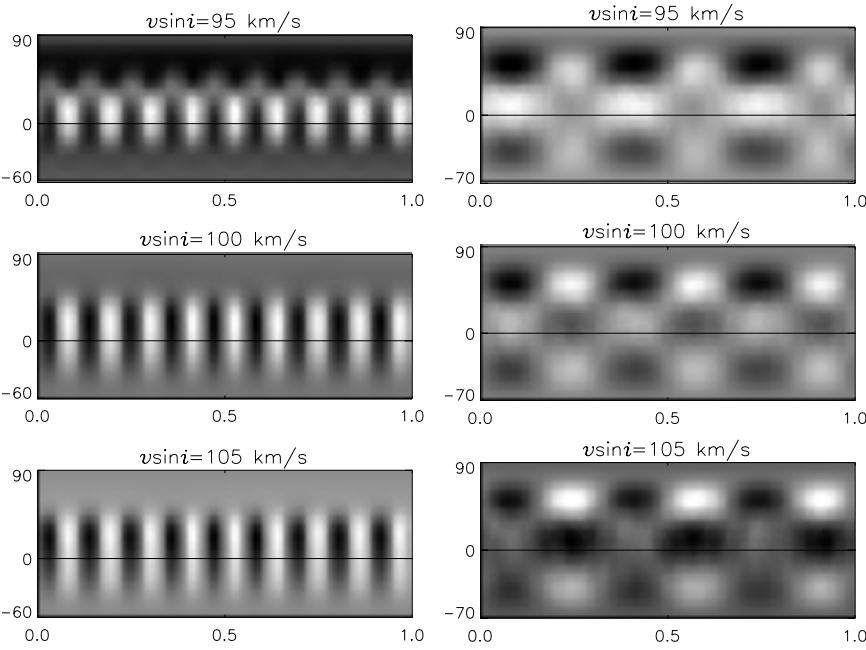

$v \sin i=100 \mathrm{~km} / \mathrm{s}$
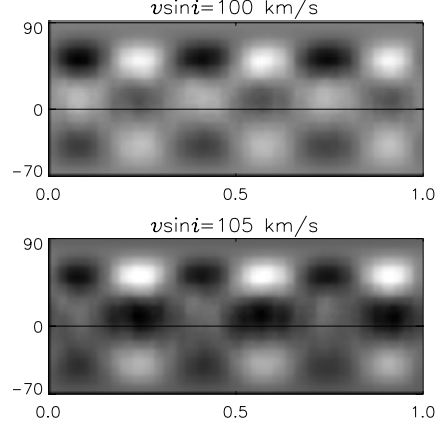

Fig. 8. The images of the Sets 2 and 6 (left and right) recovered with the true rotational velocity of $100 \mathrm{~km} \mathrm{~s}^{-1}$ and two values deviating from that by $\pm 5 \mathrm{~km} \mathrm{~s}^{-1}$.

\subsection{Frequency}

For the sectoral mode (Set 2) we allowed the frequency to vary by \pm 0.1 cycle/day. This corresponds to the changes of the pattern-rotation period by $\pm 0.1 \mathrm{~h}$, which is about $0.7 \%$. The result is shown in the left column of Fig. 9. The frequency perturbation appears in the visible polar region as a pseudo-pulsation pattern with lower azimuthal number $(|m| \approx 5)$.

For the tesseral mode (Set 6) we allowed the frequency to vary by \pm 0.2 cycle/day. This corresponds to the changes of the pattern-rotation period by $\pm 0.06 \mathrm{~h}$, which is $1.25 \%$. The result is shown in the right column of Fig. 9. The frequency perturbation affects significantly the structure of the equatorial and subequatorial pulsation patterns.

We conclude that errors in the pulsation frequency influence significantly the result of inversions. Although the sectoral and tesseral modes can be still distinguished, the clearness of the pattern can be significantly destroyed. For applying the inversion technique to real data, we suggest to test a sample of frequencies and look for the most clear pattern.

\section{Conclusions}

We showed that the traditional Doppler imaging technique can be successfully used for mapping stellar non-radial p-mode pulsations in terms of temperature fluctuations. The technique results in an image of the stellar surface in which sectoral and tesseral modes can be distinguished and the pulsational degree $\ell$ and the azimuthal order $|m|$ can be determined.

We found that sectoral and tesseral modes with $\ell-|m|=2$ (or any even number), which are symmetric with respect to the equator, can be successfully restored under various conditions. Temperature fluctuations produce a regular pattern of hot and cool regions. Strong velocity fluctuations, interpreted in terms of temperature fluctuations, result in a pattern with broad cool regions and narrow hot regions. This may serve as a diagnostics of strong velocity fields in NRP.
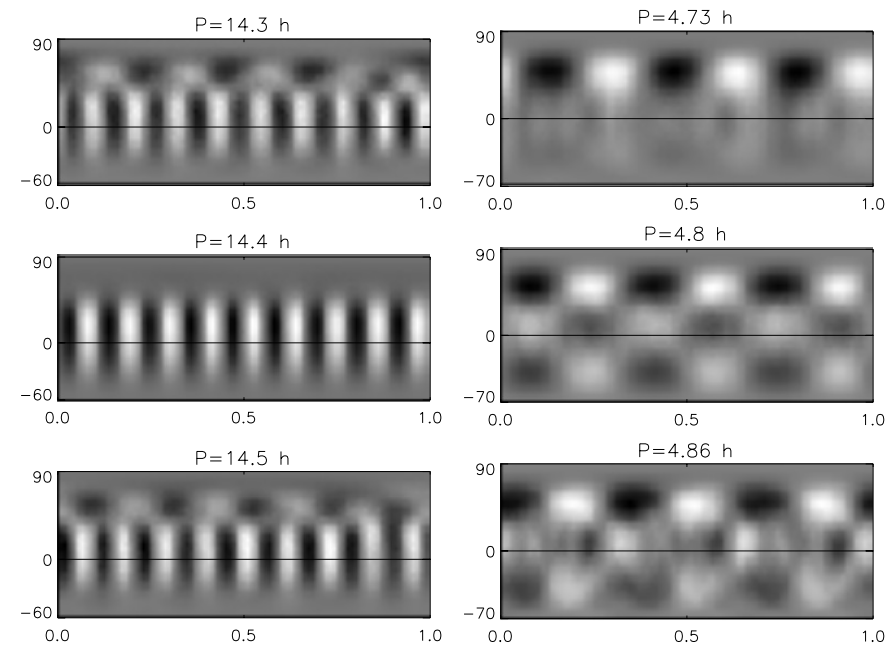

$\mathrm{P}=4.86 \mathrm{~h}$

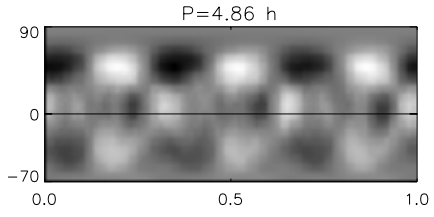

Fig. 9. The images of the Sets 2 and 6 (left and right) recovered with the true pulsation frequencies (in the middle row) and two values deviating from those.

Difficulties arise when pulsation modes are antisymmetric with respect to the equator, i.e. when $\ell-|m|=1$ or any odd number. In such modes one node line coincides with the equator, and opposite sign pulsation fluctuations appear at almost equivalent Doppler shifts in either sides of the equator. At higher inclinations, this cancels out line profile variations and no information can be recovered. At lower inclinations, the $l p v$ are rather significant, but only the azimuthal number $|m|$ can be successfully determined. A reflection pattern in the recovered image may suggest for the presence of an antisymmetric tesseral mode. Note that in symmetric modes such a reflection is hidden in the symmetric pattern.

We tested the influence of errors in the inclination of the rotational axis to the line of sight, rotational velocity and pulsation frequency. We found that variations in the first two parameters do not disturb significantly the pulsation pattern and, thus, do not influence the mode identification. This is in contrast with the effect of errors in the frequency (or pattern-rotation period), which appeared to be critical for the inversions.

We conclude that stellar surface imaging represents a novel technique for the pulsation mode identification that is complementary to other Doppler-imaging mode-identification methods. Surface imaging might especially aid in the determination of $|m|$ for rotating stars. We present an application of the surface imaging method on the bright early-type B star $\omega^{1}$ Sco in a separate paper (Berdyugina et al. 2003).

The present surface imaging technique is to be further developed for recovering the velocity field of NRP along with the temperature fluctuations. This will allow for studying physical properties of pulsations and constraining their models. Also, it will make it possible to draw some constraints on the photometric variability of pulsating stars and compare them with observations.

\section{References}

Aerts, C., \& Eyer, L. 2000, in Delta Scuti and Related Stars, ed. M. Breger, \& M. Montgomery, ASP Conf. Ser., 210, 113 
Balona, L. A. 2000, MNRAS, 319, 606

Berdyugina, S. V. 1991, Izv. Krymsk. Astrofiz. Obs., 83, 102

Berdyugina, S. V. 1998, A\&A, 338, 97

Berdyugina, S. V., Berdyugin, A. V., Ilyin, I., \& Tuominen, I. 1998, A\&A, 340, 437

Berdyugina, S. V., Berdyugin, A. V., Ilyin, I., \& Tuominen, I. 1999, A\&A, 350, 626

Berdyugina, S. V., Telting, J. H., Korhonen, H., \& Schrijvers, C. 2003, A\&A, 406, 281

Deutsch, A. 1958, in Electromagnetic Phenomena in Cosmological Physics, ed. B. Lehnert, IAU Symp. 6 (Dordrecht: Reidel), 209

Deutsch, A. 1970, AJ, 159, 985

Falk, A. F., \& Wehlau, W. H. 1974, AJ, 192, 409

Gies, D. R., \& Kullavanijaya, A. 1988, ApJ, 326, 813
Goncharsky, A. V., Stepanov, V. V., Khokhlova, V. L., \& Yagola, A. G. 1977, Sov. Astron. Lett., 3, 147

Kurucz, R. L. 1993, Kurucz CD No. 13

Mantegazza, L. 2000, in Delta Scuti and Related Stars, ed. M. Breger, \& M. Montgomery, ASP Conf. Ser., 210, 138

Piskunov, N.E., Tuominen, I., \& Vilhu, O. 1990, A\&A, 230, 363

Schrijvers, C., \& Telting, J. H. 1999, A\&A, 342, 453

Schrijvers, C., Telting, J. H., Aerts, C., Ruymaekers, E., \& Henrichs, H. F. 1997, A\&AS, 121, 343

Telting, J. H. 2003, in Asteroseismology Across the HR Diagram, ed. M. J. Thompson, M. S. Cunha, \& M. J. P. F. G. Monteiro (Kluwer) Telting, J. H., \& Schrijvers, C. 1997, A\&A, 317, 723

Vogt, S. S., \& Penrod, G. D. 1983, ApJ, 275, 661

Vogt, S. S., Penrod, G. D., \& Hatzes, A. P. 1987, ApJ, 321, 496 